\title{
Multi-modal Analytical Insights Into Li-Ion Battery Ageing with XFC
}

Peter M. Attia ${ }^{1}, \underline{\text { Stephen T. Kelly }}{ }^{2 *}$, William Harris ${ }^{2}$, Jerry Liao ${ }^{1}$, William Huang ${ }^{1}$, Yi Cui ${ }^{1}$, William C. Chueh $^{1}$

1. Department of Materials Science and Engineering, Stanford University, Stanford, CA, USA

2. Carl Zeiss Microscopy, Inc, Pleasanton, CA, USA

* Corresponding author: steve.kelly@zeiss.com

Microstructural changes with ageing in Li-ion batteries are both poorly understood and a major cause for loss of battery performance. In a large-scale application such as an electric vehicle (EV), these aging modes can lead to costly repairs and poor reliability. Furthermore, extreme fast charging (XFC) is a desirable feature for applications such as EVs, but XFC conditions generally lead to accelerated cell ageing [1-3]. To enable XFC for batteries in EVs and other critical applications, the ageing mechanisms associated with it must be better understood.

We have examined the effects of fast charging on $18650 \mathrm{LFP} /$ graphite cells charged at $5 \mathrm{C}$, where $\mathrm{C}$ rate is defined as the current required to fully charge in 1 hour. Since graphite changes color upon lithiation, optical microscopy under an inert atmosphere can detect degradation patterns throughout the electrode [4-6]. Once the cells have reached $80 \%$ of their nominal capacity ( $80 \%$ state-of-health), we perform a final slow charge prior to cell disassembly to reveal the active and inactive regions of the graphitic electrode. While pristine electrodes appear uniformly gold (i.e. fully healthy), distinct regions appear in the disassembled electrodes after XFC (Fig 1a-b). In the cycled electrode, the central portion of the electrode remains active, while darker inactive regions are localized at the periphery of the electrode. This inactive material significantly reduces the charge capacity of the cell and is not found in cells charged at slower rates, nor for cells that experienced slow charging and rapid discharging. Notably, the width of the inactive region throughout the electrode is identical to that of the current-collecting tab, suggesting that a slight compression of the electrode in the 18650 can lead to significant electrode-scale heterogeneities. Closer examination of both the active and inactive regions using scanning electron microscopy (SEM) (Fig 1c-d) reveals a distinct layer covering the surface of the inactive material. SEM images were collected after brief $(<30 \mathrm{~s})$ air exposure.

By analyzing samples from both the active and inactive regions with $\mathrm{x}$-ray nanotomography (Fig 1e-f), we observe that the surface layer of the inactive region extends $\sim 10 \mu \mathrm{m}$ into the electrode and is localized at the electrode/seperator interface. No corresponding layer was found within the anode thickness or at the electrode/current collector interface. The sample from the active region shows no sign of the additional layer throughout its thickness. X-ray nanotomography samples were prepared from small sections of electrode. The current collector was peeled away to expose the bare electrode, and the samples were imaged in air using a ZEISS Xradia 810 Ultra x-ray microscope.

To characterize the chemical composition of this inactivating surface layer, additional analysis of inactive material surface layer samples using cryogenic scanning transmission electron microscopy with electron energy loss spectroscopy (cryo-STEM-EELS) reveals the surface layer to be a growth of solidelectrolyte interphase (SEI) at the anode surface. Cryo-STEM-EELS measurements were performed on a sample extracted from the inactive region under inert conditions and transferred to the microscope without reaction to air. 
These results indicate that battery components that are generally overlooked, such as the electrode tab, may be key contributors to battery failure. Overall, this work highlights the unique, heterogeneous degradation modes during XFC and the multifaceted characterization approach required for furthering our understanding of these complex phenomena.

\section{References:}

[1] Keil, P. \& Jossen, A., Journal of Energy Storage 6, (2016) p. 125-141.

[2] Ahmed, S. et al., Journal of Power Sources 367, (2017) p. 250-262.

[3] Yang, X.-G., Leng, Y., Zhang, G., Ge, S. \& Wang, C.-Y., Journal of Power Sources 360, (2017) p. $28-40$.

[4] Harris, S. J., Timmons, A., Baker, D. R. \& Monroe, C., Chemical Physics Letters 485, (2010) p. 265-274.

[5] Ferguson, T. R. \& Bazant, M. Z., Acta 146, (2014) p. 89-97.

[6] Guo, Y. et al., The Journal of Physical Chemistry Letters 7, (2016) p. 2151-2156.
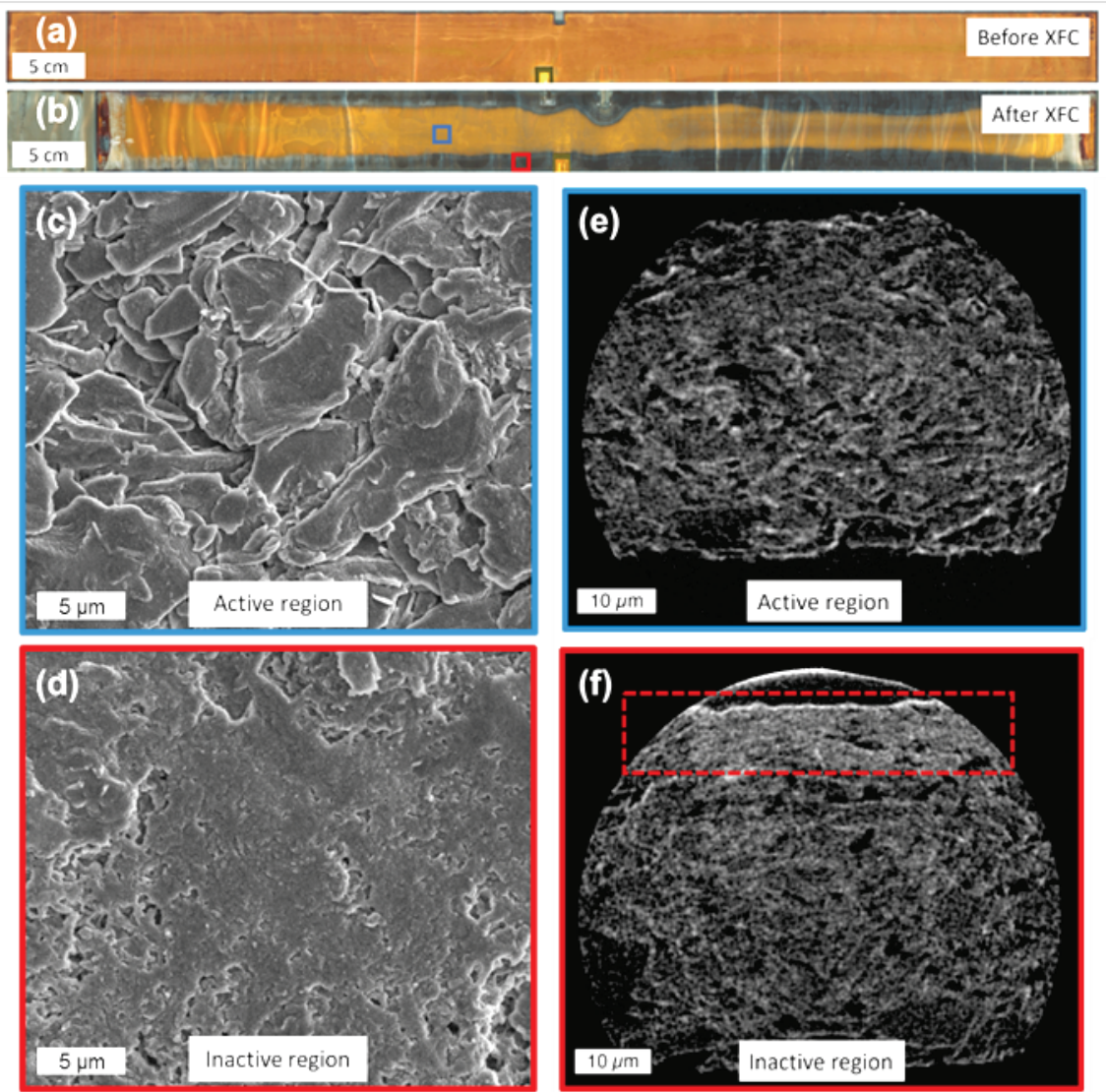

Figure 1. optical micrographs of the anode surface before (a) and after (b) XFC. Scanning electron micrographs of the anode surface from the active (c) and inactive (d) regions of the cycled electrode. Xray nanotomograms through the thickness of samples from the active (e) and inactive (f) regions of the electrode. The dotted red rectangle in (f) indicates the location of the layer forming at the anode surface. 